\title{
Quantification of Plasmodium knowlesi versus Plasmodium falciparum in the rhesus liver: implications for malaria vaccine studies in rhesus models
}

\author{
Melanie J. Shears ${ }^{1,2}$, Annette M. Seilie ${ }^{1,2}$, B. Kim Lee $\operatorname{Sim}^{3}$, Stephen L. Hoffman ${ }^{3}$ and Sean C. Murphy ${ }^{1,2,4^{*}}$ (D)
}

\begin{abstract}
Background: Rhesus macaques are valuable pre-clinical models for malaria vaccine development. The Plasmodium knowlesi/rhesus and Plasmodium falciparum/rhesus models are two established platforms for malaria vaccine testing, and both have previously been used to assess live-attenuated sporozoite vaccines. However, there is evidence that the susceptibility of the rhesus liver to P. knowlesi versus P. falciparum sporozoites likely differs, potentially complicating comparisons between these two platforms.
\end{abstract}

Methods: To quantify the differing susceptibility of rhesus to $P$. knowlesi and $P$. falciparum sporozoites, animals were infected by direct venous inoculation of purified, cryopreserved wild-type P. knowlesi sporozoites (PkSPZ) or P. falciparum sporozoites (PfSPZ). The entire liver was collected 5 days post-infection, and parasite burden in each liver lobe was quantified using an ultrasensitive Plasmodium 185 rRNA RT-PCR biomarker assay. The potential of using 185 rRNA copy number in the rhesus liver to directly measure the efficacy of vaccines targeting $P$. falciparum sporozoites and liver stages was also theoretically evaluated.

Results: Infection of rhesus with a high dose of PkSPZ led to consistently high burden liver stage infections (range 9.5-10.1 $\log _{10}$ copies $18 \mathrm{~S}$ rRNA/g of liver), with similar amounts of parasite $18 \mathrm{~S} r R N A$ detected in every liver lobe. Inoculation of rhesus with high doses of PfSPZ led to more variable, lower liver burdens (range 4.9-6.6 $\log _{10}$ copies $18 \mathrm{~S}$ rRNA/g of liver in infected lobes), with parasite $18 \mathrm{~S}$ rRNA below the limit of detection in some liver lobes. The low signal and heterogeneity of liver burden in the PfSPZ-infected animals indicates that even this extremely sensitive molecular assay cannot be used to assess reliably vaccine efficacy in the P. falciparum/rhesus platform.

Conclusions: Detection of $18 \mathrm{~S}$ rRNA in the liver following high dose intravenous PfSPZ confirmed that rhesus are modestly susceptible to wild-type P. falciparum sporozoites. However, comparison of $18 \mathrm{~S}$ rRNA RT-PCR biomarker signal indicates that the P. falciparum liver burden was 3-5 logs lower than in PkSPZ-infected animals. Quantification of this difference in liver stage burden will help guide and interpret data from pre-clinical studies of live-attenuated sporozoite vaccines in rhesus models.

Keywords: Rhesus macaque, P. falciparum, P. knowlesi, Sporozoite, Vaccine, PfSPZ, PkSPZ

*Correspondence: murphysc@uw.edu

${ }^{1}$ Department of Laboratory Medicine and Pathology, University of Washington, 750 Republican St., F870, Seattle, WA 98109, USA Full list of author information is available at the end of the article

\section{Background}

Malaria control efforts would benefit enormously from development of a highly effective and easily deployable malaria vaccine that achieves durable sterile protection

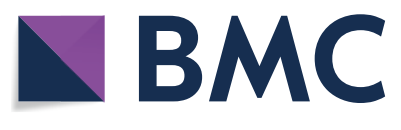

(c) The Author(s) 2020. This article is licensed under a Creative Commons Attribution 4.0 International License, which permits use, sharing, adaptation, distribution and reproduction in any medium or format, as long as you give appropriate credit to the original author(s) and the source, provide a link to the Creative Commons licence, and indicate if changes were made. The images or other third party material in this article are included in the article's Creative Commons licence, unless indicated otherwise in a credit line to the material. If material is not included in the article's Creative Commons licence and your intended use is not permitted by statutory regulation or exceeds the permitted use, you will need to obtain permission directly from the copyright holder. To view a copy of this licence, visit http://creativeco mmons.org/licenses/by/4.0/. The Creative Commons Public Domain Dedication waiver (http://creativecommons.org/publicdomain/ zero/1.0/) applies to the data made available in this article, unless otherwise stated in a credit line to the data. 
against Plasmodium falciparum malaria. The Plasmodium sporozoite and liver stages are considered strategic targets for intervention, and a number of strategies designed to block these stages have been developed including protein subunit vaccines, viral-vectored vaccines, and live-attenuated vaccines such as radiation-attenuated sporozoites and genetically- or chemically-attenuated parasite vaccines [1].

Despite numerous protective vaccine candidates being characterized in rodent malaria models, translating such findings to humans has been difficult and many clinical trials have shown underwhelming efficacy [2-4], suggesting that rodent models may not faithfully predict vaccine efficacy in humans. To date, purified, cryopreserved, live-attenuated $P$. falciparum sporozoite (PfSPZ) vaccines have provided the best vaccine efficacy in humans, achieving up to $100 \%$ sterile protection against controlled human malaria infections in clinical trials [5-7]. However, although there has been significant progress in this area [8], to achieve the goals of durable, high level vaccine efficacy at low cost, there is still a need to develop new malaria vaccine strategies and translationally-relevant models for their evaluation.

Rhesus macaques are valuable pre-clinical models that allow for assessment of malaria vaccine safety, immunogenicity and protective efficacy. Rhesus are considered excellent models of the human immune system [9], and the availability of immunological tools, together with the ability to study the liver and other tissues inaccessible in humans, makes them ideally suited for translationally-relevant vaccine research [10]. Both Plasmodium knowlesi and P. falciparum have been used in rhesus for malaria vaccine testing. Plasmodium knowlesi parasites naturally infect non-human primates and cause zoonotic malaria infections in humans [11]. The $P$. knowlesi/rhesus model can be used to evaluate malaria vaccine immunogenicity and efficacy, and because $P$. knowlesi is highly virulent in rhesus, provides a stringent model for assessing vaccine strategies prior to human trials $[11,12]$. The $P$. falciparum/rhesus model is an alternative platform that allows direct evaluation of vaccines targeting this parasite species. However, since rhesus do not support P. falciparum blood stage infections [13], this platform is typically used to assess vaccine immunogenicity as animals are not amenable to challenge with a blood stage efficacy endpoint $[14,15]$.

For live-attenuated sporozoite vaccines against $P$. falciparum, which almost certainly rely on successful sporozoite invasion in the liver for antigen delivery [15], the use of rhesus for vaccine assessment critically hinges on their susceptibility to sporozoite infection. Fortunately, despite being unable to support the P. falciparum blood stage, previous data have demonstrated that rhesus have some susceptibility to $P$. falciparum sporozoite infection in the liver. Data from two independent studies have shown that $P$. falciparum sporozoites can invade and develop in rhesus primary hepatocytes in vitro, albeit with less efficiency than $P$. falciparum in human hepatocytes or $P$. knowlesi in rhesus hepatocytes $[15,16]$. These findings validated the use of rhesus for testing the immunogenicity of live-attenuated PfSPZ vaccines, and enabled pivotal non-human primate studies [15] that guided subsequent clinical trials $[5,17]$. Complementary studies in the $P$. knowlesi/rhesus challenge model contributed further insight into the immunogenicity of live-attenuated sporozoite vaccines [18] and allowed for more definitive identification of correlates of protection in the liver [19]. Rhesus have thus been key for elucidating mechanisms of vaccine-induced immunity that are expected to translate to humans. However, integrating data from these two platforms would undoubtedly be aided by a greater understanding of rhesus susceptibility to $P$. falciparum versus $P$. knowlesi sporozoites in vivo.

Recent data are beginning to shed light on this question. In a prior study, rhesus were inoculated intravenously with a high dose $\left(6.5 \times 10^{6}\right)$ of freshly-dissected wild-type $P$. falciparum sporozoites and parasite liver burden was quantified 3 or 6 days later using ultra-sensitive 18S rRNA RT-PCR [20]. Parasite 18S rRNA was detected in the rhesus liver at both time points, with RNA copy number increasing 100-fold between days 3 and 6 [20]. This demonstrated measurable P. falciparum liver burden could be supported in rhesus, and provided the first quantification of rhesus susceptibility to $P$. falciparum sporozoites in vivo. These data also suggested the possibility of quantifying $P$. falciparum $18 \mathrm{~S}$ rRNA in the rhesus liver to directly measure efficacy of vaccines targeting sporozoites and liver stages. Here, this study aims to extend upon this previous work by directly comparing the susceptibility of rhesus to wild-type P. falciparum versus $P$. knowlesi sporozoites in vivo, and theoretically evaluating the potential to use measurements of $P$. falciparum $18 \mathrm{~S}$ rRNA in the liver as a vaccine efficacy endpoint.

\section{Methods}

\section{Study design and rationale}

Juvenile male and female Indian origin rhesus macaques (Macaca mulatta) were group-housed at the Washington National Primate Research Center (WaNPRC). Animals were malaria-naïve and tested at baseline for $\mathrm{C}$-reactive protein (CRP) and complete blood count (CBC) to evaluate their level of systemic immune activation and overall health. Individuals were randomized into three treatment groups by sex and weight. All animals were infected by direct venous inoculation (DVI) of cryopreserved 
sporozoites at day 0 . The dose and species of sporozoite used differed by treatment group as follows: Group A-1 $\times 10^{6}$ aseptic, purified, cryopreserved wild-type P. falciparum sporozoites (PfSPZ; two animals), Group B $-5 \times 10^{6}$ PfSPZ (two animals), and Group C-0.5 $\times 10^{6}$ purified cryopreserved, wild-type $P$. knowlesi sporozoites (PkSPZ; two animals). Sporozoite doses were chosen to reflect high doses based on published studies [20], with the final number determined by sporozoite availability. Animals were humanely euthanized at day 5 post challenge, at precisely $120 \mathrm{~h}$ post-challenge to the hour. This time was selected as it was expected to reliably precede parasite egress from the liver $[21,22]$. The number of animals used was driven by budget constraints; the study was not designed for statistical power and, because of this, statistical tests are not reported between groups.

\section{Cryopreserved sporozoites}

PfSPZ and PkSPZ were produced by Sanaria as described $[5,6]$. Both were purified and cryopreserved, and the PfSPZ were aseptic. Sanaria's aseptic, purified, cryopreserved PfSPZ have been shown to cause asexual erythrocytic stage infection in $100 \%$ of malaria-naïve human subjects at a dose of $3.2 \times 10^{3}$ by DVI [6]. Purified, cryopreserved PkSPZ have been shown to cause asexual erythrocytic stage infection in $100 \%$ of malarianaive rhesus macaques at a dose of $0.5 \times 10^{3}$ by DVI (S. Chakravarty, unpublished). PfSPZ were vialed at a $1 \times 10^{6}$ PfSPZ per vial, and PkSPZ were vialed at $5 \times 10^{4} \mathrm{PkSPZ}$ per vial. Vials from a single PfSPZ batch and single PkSPZ batch were used. Separate vials of PfSPZ or vials of PkSPZ were used for each animal. PfSPZ and PkSPZ products were shipped, stored, thawed, diluted, and administered in strict accordance with Sanaria protocols. Sporozoites were diluted into $1 \%$ human serum albumin (CSL-Behring, \#44206-251-10) in sterile USP grade phosphate buffered saline (RMBio, \#BSS-PBS-1X6). Direct venous inoculation of diluted sporozoites was performed using a syringe into the saphenous vein under ketamine and dexmedetomidine sedation. Correct entry of the syringe into the vein was confirmed by pulling back on the syringe plunger to observe a return of blood prior to administration of the sporozoites.

\section{Blood and liver tissue processing}

Blood for Plasmodium 18S rRNA RT-PCR was drawn prior to euthanasia into ETDA tubes. Blood was stored briefly at room temperature then $1 \mathrm{~mL}$ was added to $19 \mathrm{~mL}$ of NucliSENS lysis buffer (bioMérieux, \#28013) and mixed to achieve homogeneous lysis. Liver tissue was collected after euthanasia into RPMI media (Gibco, \#22400) supplemented with Penicillin-StreptomycinGlutamine (Gibco, \#10378-016) and 10\% heat-inactivated fetal bovine serum (Sigma, \#4135). The gall bladder was removed by dissection, and the livers were divided into four lobes as follows: left lateral lobe (LLL), left medial lobe (LML), right medial lobe plus caudate lobe $(\mathrm{RML}+\mathrm{C})$, and right lateral lobe (RLL). Lobes were individually weighed, then separately homogenized in $100 \mathrm{~mL}$ of NucliSENS lysis buffer with an immersion blender. Multiple immersion blenders were used with bleach and ethanol cleanings to eliminate carryover between animals. Lysed blood and liver homogenates were stored at $-80^{\circ} \mathrm{C}$ until extraction.

\section{Nucleic acid extraction and Plasmodium 18S rRNA RT-PCR}

Lysed blood and total liver lobe homogenates were thawed and further diluted for a final concentration of 50 $\mu \mathrm{L}$ whole blood or $50 \mathrm{mg}$ liver to $2 \mathrm{~mL}$ of lysis buffer, of which $1 \mathrm{~mL}$ of lysate was processed in duplicate by the Abbott m2000sp. using the mSample RNA preparation kit (Abbott Molecular). Extracted RNA were subject to Plasmodium 18S rRNA quantitative reverse transcription-PCR (qRT-PCR) using the SensiFAST ${ }^{\mathrm{TM}}$ Probe LoRox One-Step Kit (Bioline \#BIO-78005) as previously described [23] but with locked nucleic acid analogs [+_] on the Pan-Plasmodium primers (Forward PanDDT1043F19: AAAGTTA[+A]GGGA[+G][+T]GAAGA, Reverse PanDDT1197R22: AA[+G]ACTTTGATT TCTC[+A]TAAGG; Qiagen) in addition to a quencher modification on the $P$. falciparum probe (5'-[6-FAM]ATTTATTCAGTAATCAAATTAGGAT-3' [Black Hole Quencher 1 PLUS]; LGC BioSearch Technologies) to permit an increased annealing temperature of $54{ }^{\circ} \mathrm{C}$ for improved assay specificity. Each specimen was quality controlled with a TATA-Binding Protein mRNA RT-PCR, and each qRT-PCR run was monitored with well-characterized run controls consisting of human whole blood samples. Results were quantified using an Armored RNA calibrator standard that encodes the P. falciparum $18 \mathrm{~S}$ rRNA (Asuragen).

\section{Results}

The study was designed to directly compare the susceptibility of the rhesus liver to wild-type $P$. falciparum and P. knowlesi sporozoites in vivo (Fig. 1). Blood was drawn from animals at baseline for CRP and $\mathrm{CBC}$ to evaluate their health, and animals were inoculated at day 0 by DVI of either $P$. falciparum and $P$. knowlesi sporozoites according to their treatment group. Two animals per group were inoculated with sporozoites as follows: Group A-1 $\times 10^{6}$ PfSPZ, Group B- $5 \times 10^{6}$ PfSPZ, Group C- $0.5 \times 10^{6}$ PkSPZ. Groups A and B were designed to assess the dose-dependence of $P$. falciparum $18 \mathrm{~S}$ rRNA liver burden following sporozoite inoculation, and to identify if either dose would theoretically allow for measurement 


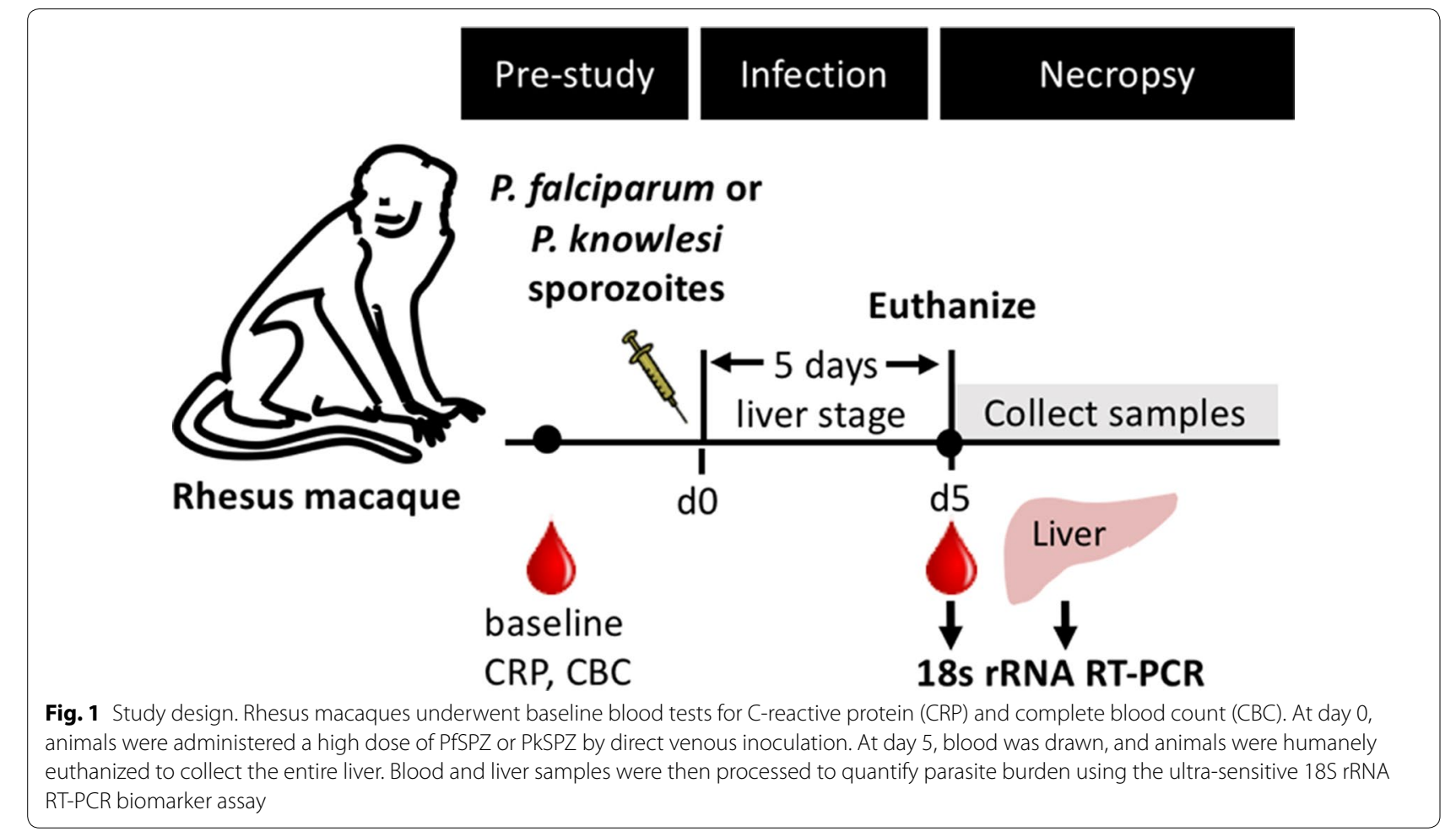

of parasite 18S rRNA in the liver as a potential vaccine efficacy endpoint. Group $C$ was designed to be a comparator, using the highest feasible dose of $P$. knowlesi sporozoites based on the PkSPZ vialling amount. At exactly 5 days post-sporozoite inoculation, blood was collected for 18S rRNA RT-PCR analysis to assess if parasites had egressed from the liver. Immediately thereafter, animals were euthanized, and livers collected, divided into four lobes, then processed and analyzed to determine parasite burden in each lobe using a slightly modified version of a well-established $18 \mathrm{~S}$ rRNA RT-PCR biomarker assay [20, 23].

The results for parasite burden in the liver are expressed as $\log _{10}$ copies $18 \mathrm{~S}$ rRNA per $g$ of tissue (Fig. 2a and Table 1). Three of the four animals inoculated with PfSPZ had measurable parasite 18S rRNA in the liver at 5 days post-infection, indicating $P$. falciparum liver burdens following sporozoite administration were somewhat unreliable. The animal with undetectable parasite $18 \mathrm{~S}$ rRNA had received a dose of $1 \times 10^{6} \mathrm{PfSPZ}$. The other animal that received this dose had detectable parasite $18 \mathrm{~S}$ rRNA in every liver lobe, with a mean of $5.57 \log _{10}$ copies per $\mathrm{g}$ of liver. Both animals that received the higher dose of $5 \times 10^{6} \mathrm{PfSPZ}$ had detectable parasite $18 \mathrm{~S}$ rRNA in the liver, suggesting some impact of sporozoite dose on assay outcome. One animal in this group had 18S rRNA signal in two of four liver lobes, with a mean of 5.32 $\log _{10}$ copies per $g$ of liver in the infected lobes, while the other animal had parasite 18S rRNA in every lobe with a mean of $6.27 \log _{10}$ copies per $g$ of liver. Both animals that received PkSPZ had much higher liver parasite $18 \mathrm{~S}$ rRNA concentrations that were consistent across all four lobes, with means of 9.52 and $9.72 \log _{10}$ copies per g of liver. Parasite $18 \mathrm{~S}$ rRNA copy number was thus $3-5 \log$ higher in animals that received PkSPZ versus PfSPZ, despite the PkSPZ dose being 2-10 fold lower than in the PfSPZ groups.

The magnitude of parasite 18S rRNA in the liver did not appear to correlate with baseline CRP levels or animal body weight (Table 1). While the small sample size of this study precluded any formal correlation analysis, qualitative assessment of the data suggested that these factors were not likely confounding variables. Specifically, for CRP levels, although animals had a range of baseline CRP levels, there was no apparent correlation between this and assay outcome. For body weight, although the smallest animal had the highest $18 \mathrm{~S}$ rRNA signal following PkSPZ inoculation, the largest animal had the highest 18S rRNA signal following PfSPZ inoculation, giving no clear relationship between body weight and signal. Similarly, for CBC measures, all animals had blood cell counts that were within normal limits based on their age and experience of veterinary staff at the primate center. By contrast, preliminary observations suggest there may be a potential correlation between sex and liver parasite burden following PfSPZ infection. This was evident as both 


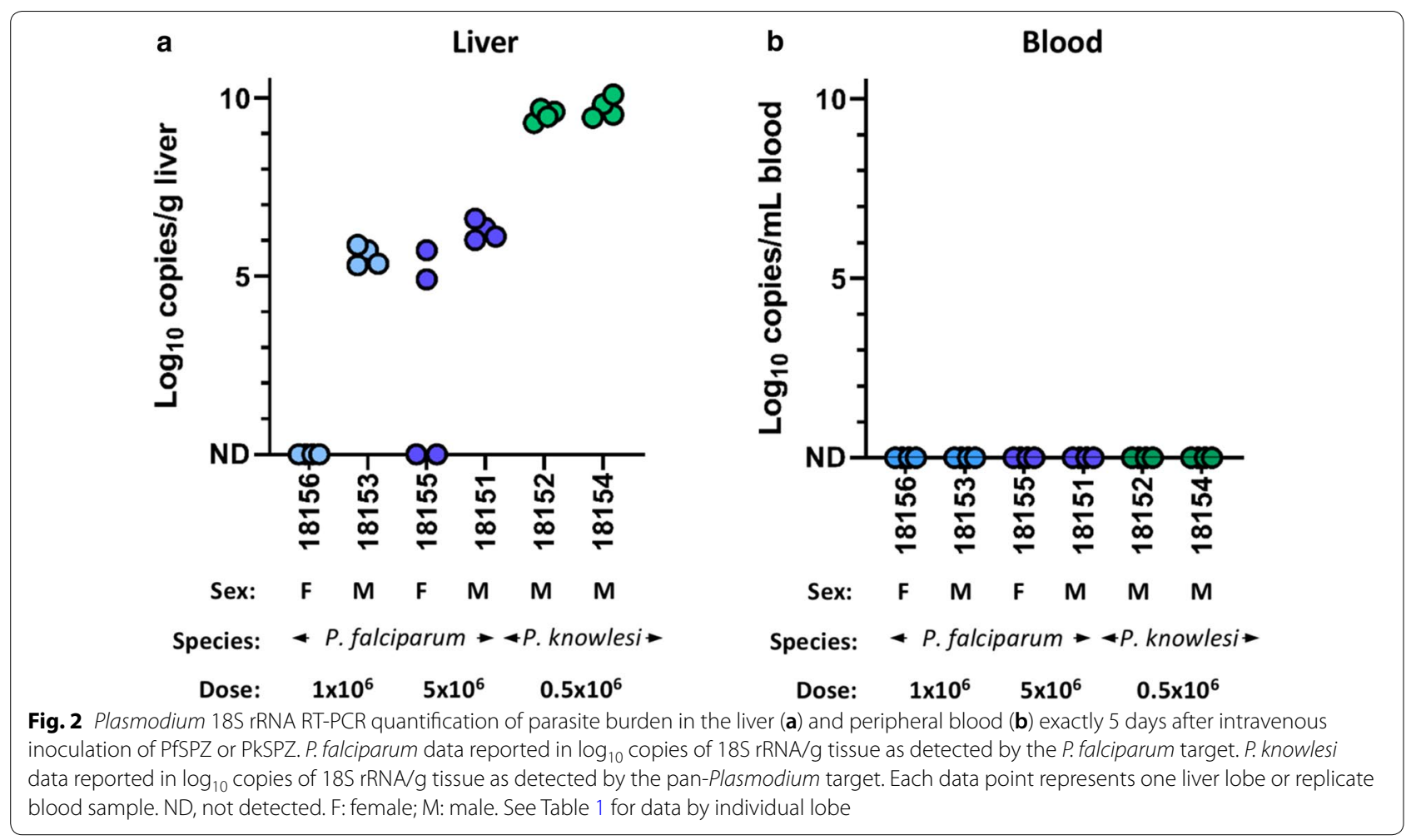

Table 1 18S rRNA quantification of liver parasite burden by lobe following intravenous inoculation of PfSPZ or PkSPZ

\begin{tabular}{lllllllllll}
\hline Animal & Group & LLL & LML & RLL & RML+C & Avg $^{*}$ & Age & Sex & Weight $(\mathbf{k g})$ & CRP $(\mathbf{m g} / \mathbf{L})$ \\
\hline A18156 & P. falciparum $\left(1 \times 10^{6}\right)$ & N.D & N.D & N.D & N.D & N.D & 1 year 6 months & Female & 2.7 & 0.5 \\
A18153 & P. falciparum $\left(1 \times 10^{6}\right)$ & 5.31 & 5.73 & 5.35 & 5.88 & 5.57 & 1 year 6 months & Male & 2.35 & $<0.2$ \\
A18155 & P. falciparum $\left(5 \times 10^{6}\right)$ & N.D & N.D & 5.73 & 4.91 & 5.32 & 1 year 6 months & Female & 2.4 & 0.3 \\
A18151 & P. falciparum $\left(5 \times 10^{6}\right)$ & 6.02 & 6.35 & 6.11 & 6.61 & 6.27 & 1 year 6 months & Male & 2.9 & 0.2 \\
A18152 & P. knowlesi $\left(0.5 \times 10^{6}\right)$ & 9.69 & 9.30 & 9.61 & 9.47 & 9.52 & 1 year 6 months & Male & 3.15 & 0.2 \\
A18154 & P. knowlesi $\left(0.5 \times 10^{6}\right)$ & 10.09 & 9.53 & 9.82 & 9.45 & 9.72 & 1 year 7 months & Male & 1.95 & 0.7 \\
\hline
\end{tabular}

P. falciparum data reported in $\log _{10}$ copies of $18 \mathrm{~S} \mathrm{rRNA} / \mathrm{g}$ tissue as detected by the $P$. falciparum target. $P$. knowlesi data reported in $\log _{10}$ copies of $18 \mathrm{~S}$ rRNA/g tissue as detected by the Pan Plasmodium target

$L M L$ left medial lobe, $R L L$ right lateral lobe, LLL left lateral lobe, $R M L+C$ right medial lobe and caudate lobe, CRP C-reactive protein, N.D not detected, $F$ female, $M$ male, Avg* mean of samples where Plasmodium $18 \mathrm{~S}$ rRNA was detected

males receiving PfSPZ had detectable $18 \mathrm{~S}$ rRNA in every liver lobe, but females in the equivalent treatment groups either had no detectable $18 \mathrm{~S}$ rRNA or signal in only some liver lobes.

The results for parasite burden in the blood are expressed as $\log _{10}$ copies $18 \mathrm{~S}$ rRNA per $\mathrm{mL}$ of whole blood (Fig. 2b). Regardless of the treatment group, no animal was found to have detectable parasite $18 \mathrm{~S}$ rRNA in the blood at 5 days post sporozoite inoculation. This was consistent with the known duration of the $P$. falciparum liver stage in humans and the $P$. knowlesi liver stage in rhesus [21, 22], and the previous data showing no $P$. falciparum $18 \mathrm{~S}$ rRNA was detectable in the blood of rhesus 6 days post-sporozoite inoculation [20]. This demonstrated that despite the high sporozoite doses given, no detectable parasites had egressed from the liver into the blood at the time of euthanasia and liver collection. Thus, the entirety of the parasite $18 \mathrm{~S}$ rRNA biomarker signal in the liver as measured above was determined to have originated from parasites in the liver tissue. 


\section{Discussion}

Previous studies with the $P$. falciparum/rhesus and $P$. knowlesi/rhesus platforms have demonstrated the value of these models for assessing live-attenuated sporozoite vaccines and elucidating mechanisms of vaccine-induced immunity relevant to humans [15, 17-19]. Since these live-attenuated vaccines must rely on sporozoite invasion for antigen delivery to the liver, studies across these two platforms have only been possible because rhesus have demonstrated some susceptibility to $P$. falciparum sporozoites $[15,16]$. However, despite evidence that rhesus are less susceptible to $P$. falciparum versus $P$. knowlesi sporozoite infection, their relative ability to infect and replicate in the rhesus liver has never been directly compared in vivo. Here, using an ultra-sensitive Plasmodium $18 \mathrm{~S}$ rRNA biomarker assay, parasite rRNA copy number in the liver was found to be 3-5 orders of magnitude lower in animals administered PfSPZ versus PkSPZ at 5 days post-infection. While the sample size of this study was small, it was nonetheless sufficient to identify striking trends that have significant relevance for the ongoing use of rhesus in malaria vaccine studies. These findings highlight the fact that there are likely significant differences in host-parasite interactions between $P$. falciparum and $P$. $k n o w l e s i$ in the rhesus liver, which in turn are expected to impact antigen delivery and the immunogenicity of liveattenuated sporozoite vaccines.

The most notable finding of this study is that administration of wild-type cryopreserved $P$. falciparum sporozoites (PfSPZ) resulted in inconsistent, heterogeneous liver stage burdens in vivo. Given that the PfSPZ inoculum was 300 to 1500 times greater than required for $100 \%$ blood stage infection in humans [6] and 1000 times the inoculum required to achieve $100 \%$ blood stage infection with PkSPZ in rhesus (S. Chakravarty, unpublished), this suggests that $P$. falciparum sporozoites must face considerable barriers to infection in rhesus in vivo. These barriers could include sporozoite exit from the circulation, invasion of hepatocytes, proliferation within hepatocytes, or avoiding immune clearance mechanisms in the liver [24]. The impact of each of these events cannot be distinguished with the current study design, but it is possible to make several further observations. First, although $P$. yoelii sporozoite invasion and development in vivo can be affected by cryopreservation [25], the robust infections seen in the PkSPZ comparator group suggests that cryopreservation was not responsible for the striking difference seen in liver parasite burdens between animals infected with PfSPZ versus PkSPZ in this study. Indeed, although it remains formally possible that $P$. falciparum and $P$. knowlesi sporozoites may tolerate cryopreservation to different extents, the comparable infectivity of cryopreserved PfSPZ in humans and cryopreserved PkSPZ in rhesus described above argue against this as a significant confounding variable. Similarly, as P. falciparum sporozoites can invade rhesus hepatocytes in vitro $[15,16]$, this implies that sporozoite invasion is unlikely to be completely blocked in vivo. On the other hand, as P. falciparum liver stage schizont development is only supported at low frequencies in rhesus hepatocytes in vitro [16], this argues that liver stage development may be severely impaired in vivo. Given that avoidance of immune clearance mechanisms by liver stage parasites involves complex and species-specific manipulations of the host cell [26], it is also reasonable to speculate that any $P$. falciparum liver stages that develop in the rhesus liver will have poorer survival than simian-adapted parasite species.

The identification of a potential correlation between sex and liver parasite burden following infection with PfSPZ is of considerable interest. Although there were only two females in this study, the finding that one had no detectable parasite $18 \mathrm{~S}$ rRNA, and the other had lower signal in fewer liver lobes than the male in the same group was noteworthy. Due to the small sample size of this study, it was not possible to exclude the alternate hypothesis that these findings were due to chance alone, or that despite rigorous procedures, the PfSPZ administration failed in the one animal that did not develop detectable liver stage parasite burden. However, as every effort was made to use age- and weight-matched animals, and to confirm correct administration of PfSPZ into the vein, additional studies in the future may be warranted to determine if this apparent sex-specific decrease in $P$. falciparum liver stage burden is reproducible. While comparatively little is known about sex-specific responses to primary malaria infection, there is an emerging body of literature about sex-specific responses to vaccination $[27$, $28]$. As the animals used here were juveniles, it is unlikely that any sex-specific effects are due to the influence of adult sex hormones on the immune system as documented for live-attenuated sporozoite vaccines in mice [29]. However, other sex-specific immunological differences have been described in human infants and children [28], including in response to the RTS,S malaria vaccine, indicating that the sexes begin to diverge in relevant ways prior to puberty. Given that children are one of the primary target populations for PfSPZ vaccines in endemic areas, it may be beneficial to determine if juvenile rhesus recapitulate these sex-specific differences and responses to malaria vaccination. Further studies to investigate and compare the susceptibility of juvenile versus adult rhesus may also be important to pursue. These studies would enhance the value of rhesus for malaria vaccine testing, and are expected to be key topics for future investigation.

Regardless of the underlying reasons, the heterogeneity of $P$. falciparum burden in the rhesus liver suggests 
quantification of parasite $18 \mathrm{~S}$ rRNA is unlikely to provide a useful efficacy measure for vaccines targeting sporozoites and liver stages in the P. falciparum/rhesus model. The potential of using the 18S rRNA biomarker assay for this purpose was suggested by a previous study, where rhesus macaques were intravenously administered $6.5 \times 10^{6}$ freshly-purified wild-type $P$. falciparum sporozoites, and parasite $18 \mathrm{~S}$ rRNA copy number was found to increase between animals sacrificed at days 3 and 6 [20]. This finding suggested that P. falciparum $18 \mathrm{~S}$ rRNA signal could be measured in the rhesus liver over a dynamic range, and hence, that differences in copy number between naïve and vaccinated animals might be used to quantify vaccine efficacy as routinely done in rodent malaria models [30, 31]. Since large doses of freshly-dissected $P$. falciparum sporozoites can be difficult to obtain, lower doses of cryopreserved wild-type sporozoites were assessed here as these would be more ideally suited for vaccine studies. While $P$. falciparum $18 \mathrm{~S}$ rRNA copy numbers were indeed lower in the current study consistent with the lower sporozoite dose, they were broadly concordant with the previous data, with the day 5 copy numbers measured here similar to or greater than the day 3 copy numbers in the previous study [20]. However, neither dose of PfSPZ resulted in consistent burdens, meaning that parasite $18 \mathrm{~S}$ rRNA copy number measurements would be highly subject to where in the liver was sampled, or necessitate sampling of the entire liver as performed here. Unfortunately, this may be impractical for vaccine studies since protocols for simultaneous processing of liver samples for $18 \mathrm{~S}$ rRNA and immunological assays are yet to be developed for rhesus, and costs and ethical concerns would preclude most researchers from performing immunogenicity and efficacy studies in parallel cohorts of rhesus. Taken together, this ultimately suggests that quantification of parasite $18 \mathrm{~S}$ rRNA in the liver is unlikely to be a feasible way to assess vaccine efficacy in the $P$. falciparum/rhesus model.

Finally, this study has several important implications for research involving live-attenuated parasite vaccines in rhesus. Live-attenuated vaccines that rely on sporozoite invasion of the liver encompass a spectrum of vaccine concepts that include radiation-attenuated sporozoites, late-arresting genetically-attenuated parasites, and chemically-attenuated parasites [1], the latter including the PfSPZ-CVac chemoprophylaxis vaccine [6]. These vaccines differ in their methods of attenuation, the extent of parasite development in the liver, and the corresponding breadth of antigens delivered to the immune system [1]. Given the ability to meaningfully evaluate these vaccines is critically dependent on to what extent rhesus can support sporozoite invasion and liver stage development, the quantitative data presented here offer new insight into how the $P$. falciparum/rhesus and P. knowlesi/rhesus platforms can best be used to evaluate these vaccines. For radiation-attenuated sporozoites that arrest early in the liver, previous studies have already demonstrated that both the P. falciparum and P. knowlesi platforms can provide highly valuable immunogenicity data about these vaccines $[15,17,18]$. Given the relatively poor susceptibility of rhesus to PfSPZ revealed here, it may be necessary to re-interpret some of the data from past studies of $P$. falciparum attenuated sporozoite vaccines in rhesus to take this decreased susceptibility into consideration. However, this certainly does not impact the validity of using rhesus to assess antibody and $\mathrm{T}$ cell responses induced by $P$. falciparum attenuated sporozoite vaccines, but rather serves to refine understanding of the model and potentially improve how findings from these studies are extrapolated to humans. Equally, as there is evidence that $P$. falciparum sporozoites can invade rhesus hepatocytes in vitro $[15,16]$ and in vivo [20], this also suggests that both platforms can be used to study responses to pre-formed antigens induced by radiationattenuated sporozoite vaccines [32], with the caveat that the decreased susceptibility of rhesus to $P$. falciparum sporozoites should again be taken into consideration. The decreased susceptibility of the rhesus liver to P. falciparum versus $P$. knowlesi sporozoites should also be considered for making comparisons across platforms, as it is expected to impact antigen load, antigen distribution across the liver, and the expression of liver stage-specific antigens. Conversely, for genetically- or chemicallyattenuated parasites vaccines that arrest at later stages of development, the data presented here indicate that $P$. falciparum/rhesus model is unsuitable for evaluation of these vaccines, and suggests such vaccines should instead be assessed using alternative platforms.

\section{Conclusions}

This study provides the first direct quantitative comparison of the relative susceptibility of the rhesus liver to wild-type $P$. falciparum versus $P$. knowlesi sporozoites in vivo. This data will help guide pre-clinical studies of live-attenuated parasite vaccines in the $P$. falciparum/ rhesus and $P$. knowlesi/rhesus models and enable better integration data from vaccine studies across platforms.

\section{Abbreviations}

CBC: Complete blood count; CRP: C-reactive protein; DVI: Direct venous inoculation; LLL: Left lateral lobe; LML: Left medial lobe; PCR: Polymerase chain reaction; PfSPZ: P. falciparum sporozoites; PkSPZ: P. knowlesi sporozoites; qRT-PCR: Quantitative reverse transcription PCR; RLL: Right lateral lobe; RML +C: Right medial lobe plus caudate lobe; rRNA: Ribosomal RNA; WaNPRC: Washington National Primate Research Center. 


\section{Acknowledgements}

We thank all the staff at the WaNPRC involved in this study and Sanaria Inc. for production of PfSPZ and PkSPZ.

\section{Authors' contributions}

MJS and SCM were jointly responsible for study design. MJS was responsible for sporozoite administration and coordinating all procedures that took place at the WaNPRC. BKLS and SLH contributed sporozoite products and guidance for their handling and administration. MJS, AMS and SCM were responsible for tissue handling. AMS was responsible for nucleic acid extraction and $18 \mathrm{~S}$ rRNA RT-PCR assay. MJS, AMS and SCM were responsible for data analysis. MJS was responsible for writing the manuscript with assistance from SCM, AMS and SLH. All authors reviewed the data. All authors read and approved the final manuscript.

\section{Authors' information}

SCM is a Core Scientist of the WaNPRC, the Medical Director of the Human Challenge Center at the Seattle Children's Research Institute, and an Investigator at the Seattle Malaria Clinical Trials Center. He is also the Assistant Director of the Clinical Microbiology Laboratory, Laboratory Medicine, University of Washington Medical Center. SCM, AMS and collaborators developed the Plasmodium 18S rRNA RT-PCR assay as a diagnostic tool for use in controlled human malaria infection trials, field trials, and pre-clinical research studies.

\section{Funding}

Funding was provided by NIH NIAID grants R03 Al144143 (SCM), R01 Al141857 (SCM), P51OD010425 (WaNPRC) and SBIR 2R44AI058375-09 (SLH). Production of the PfSPZ and PkSPZ by Sanaria Inc. was supported by NIH NIAID SBIR grants 4R44AI055229-08, 3R44AI055229-06S1, and 5R44AI058499-05.

\section{Availability of data and materials}

The datasets used and/or analyzed during the current study are available from the corresponding author on reasonable request.

\section{Ethics approval and consent to participate}

All procedures were conducted in accordance with an approved University of Washington Institutional Animal Care and Use Committee Protocol. All animals were cared for in accordance with the NIH Guide for the Care and Use of Laboratory Animals.

\section{Consent for publication}

Not applicable.

\section{Competing interests}

MJS, AMS and SCM declare that they have no relevant competing interests. BKLS is an employee of Sanaria Inc. SLH is Chief Executive and Scientific Officer of Sanaria Inc.

\section{Author details}

${ }^{1}$ Department of Laboratory Medicine and Pathology, University of Washington, 750 Republican St., F870, Seattle, WA 98109, USA. ${ }^{2}$ Center for Emerging and Re-emerging Infectious Diseases, University of Washington, 750 Republican St., Seattle, WA 98109, USA. ${ }^{3}$ Sanaria, Inc., 9800 Medical Center Drive, Suite A209, Rockville, MD 20850, USA. ${ }^{4}$ Washington National Primate Research Center, University of Washington, 1959 NE Pacific St., Seattle, WA 98195, USA.

\section{Received: 15 May 2020 Accepted: 13 July 2020}

Published online: 31 August 2020

\section{References}

1. Draper SJ, Sack BK, King CR, Nielsen CM, Rayner JC, Higgins MK, et al. Malaria vaccines: recent advances and new horizons. Cell Host Microbe. 2018;24:43-56.

2. RTS,S Clinical Trials Partnersip, Agnandji ST, Lell B, Soulanoudjingar SS, Fernandes JF, Abossolo BP, et al. First results of phase 3 trial of RTS, S/AS01 malaria vaccine in African children. N Engl J Med. 2011;365:1863-75.

3. White MT, Verity R, Griffin JT, Asante KP, Owusu-Agyei S, Greenwood B, et al. Immunogenicity of the RTS, S/AS01 malaria vaccine and implications for duration of vaccine efficacy: secondary analysis of data from a phase 3 randomised controlled trial. Lancet Infect Dis. 2015:15:1450-8.

4. Tiono AB, Nebie I, Anagnostou N, Coulibaly AS, Bowyer G, Lam E, et al. First field efficacy trial of the ChAd63 MVA ME-TRAP vectored malaria vaccine candidate in 5-17 months old infants and children. PLoS ONE. 2018;13:e0208328.

5. Seder RA, Chang LJ, Enama ME, Zephir KL, Sarwar UN, Gordon IJ, et al. Protection against malaria by intravenous immunization with a nonreplicating sporozoite vaccine. Science. 2013;341:1359-65.

6. Mordmuller B, Surat G, Lagler H, Chakravarty S, Ishizuka AS, Lalremruata $A$, et al. Sterile protection against human malaria by chemoattenuated PfSPZ vaccine. Nature. 2017:542:445-9.

7. Jongo SA, Church LWP, Mtoro AT, Chakravarty S, Ruben AJ, Swanson li PA, et al. Increase of dose associated with decrease in protection against controlled human malaria infection by PfSPZ Vaccine in Tanzanian adults. Clin Infect Dis. 2019:ciz1152 (Online ahead of print).

8. Richie TL, Billingsley PF, Sim BK, James ER, Chakravarty S, Epstein JE, et al. Progress with Plasmodium falciparum sporozoite (PfSPZ)-based malaria vaccines. Vaccine. 2015;33:7452-61.

9. Messaoudi I, Estep R, Robinson B, Wong SW. Nonhuman primate models of human immunology. Antioxid Redox Signal. 2011;14:261-73.

10. Phillips KA, Bales KL, Capitanio JP, Conley A, Czoty PW, Hart BA, et al. Why primate models matter. Am J Primatol. 2014;6:801-27.

11. Pasini EM, Zeeman AM, Voorberg Van Derwa A, Kocken CHM. Plasmodium knowlesi: a relevant, versatile experimental malaria model. Parasitology. 2018;145:56-70.

12. Rogers WO, Weiss WR, Kumar A, Aguiar JC, Tine JA, Gwadz R, et al. Protection of rhesus macaques against lethal Plasmodium knowlesi malaria by a heterologous DNA priming and poxvirus boosting immunization regimen. Infect Immun. 2002;70:4329-35.

13. Perkins M. Inhibitory effects of erythrocyte membrane proteins on the in vitro invasion of the human malarial parasite (Plasmodium falciparum) into its host cell. J Cell Biol. 1981;90:563-7.

14. Phares TW, May AD, Genito CJ, Hoyt NA, Khan FA, Porter MD, et al. Rhesus macaque and mouse models for down-selecting circumsporozoite protein based malaria vaccines differ significantly in immunogenicity and functional outcomes. Malar J. 2017;16:115.

15. Epstein JE, Tewari K, Lyke KE, Sim BK, Billingsley PF, Laurens MB, et al. Live attenuated malaria vaccine designed to protect through hepatic CD8(+) T cell immunity. Science. 2011;334:475-80.

16. Millet P, Collins WE, Aikawa M, Cochrane AH, Nguyen-Dinh P. Use of non-human primate hepatocytes for in vitro study of the pre-erythrocytic stages of malaria parasites. Bull World Health Organ. 1990;68(Suppl):60-5.

17. Ishizuka AS, Lyke KE, DeZure A, Berry AA, Richie TL, Mendoza FH, et al. Protection against malaria at 1 year and immune correlates following PfSPZ vaccination. Nat Med. 2016:22:614-23.

18. Weiss WR, Jiang CG. Protective CD $8+T$ lymphocytes in primates immunized with malaria sporozoites. PLoS ONE. 2012;7:e31247.

19. Pichyangkul S, Spring MD, Yongvanitchit K, Kum-Arb U, Limsalakpetch A, Im-Erbsin R, et al. Chemoprophylaxis with sporozoite immunization in $P$ knowlesi rhesus monkeys confers protection and elicits sporozoitespecific memory T cells in the liver. PLoS ONE. 2017;12:e0171826.

20. Murphy SC, Ishizuka AS, Billman ZP, Olsen TM, Seilie AM, Chang M, et al. Plasmodium 18S rRNA of intravenously administered sporozoites does not persist in peripheral blood. Malar J. 2018;17:275.

21. Shortt HE, Fairley NH, Covell G, Shute PG, Garnham PC. The pre-erythrocytic stage of Plasmodium falciparum. Trans R Soc Trop Med Hyg. 1951;44:405-19.

22. Garnham PC, Lainson R, Cooper W. The tissue stages and sporogony of Plasmodium knowlesi. Trans R Soc Trop Med Hyg. 1957;51:384-96.

23. Seilie AM, Chang M, Hanron AE, Billman ZP, Stone BC, Zhou K, et al. Beyond blood smears: qualification of Plasmodium $18 \mathrm{~S}$ rRNA as a biomarker for controlled human malaria infections. Am J Trop Med Hyg. 2019;100:1466-76.

24. Vaughan AM, Kappe SHI. Malaria parasite liver infection and exoerythrocytic biology. Cold Spring Harb Perspect Med. 2017;7:a025486.

25. Roestenberg M, Bijker EM, Sim BKL, Billingsley PF, James ER, Bastiaens GJH, et al. Controlled human malaria infections by intradermal injection of cryopreserved Plasmodium falciparum sporozoites. Am J Trop Med Hyg. 2013:88:5-13. 
26. Agop-Nersesian C, Niklaus L, Wacker R, Theo Heussler V. Host cell cytosolic immune response during Plasmodium liver stage development. FEMS Microbiol Rev. 2018:42:324-34.

27. Fischinger S, Boudreau CM, Butler AL, Streeck H, Alter G. Sex differences in vaccine-induced humoral immunity. Semin Immunopathol. 2019;41:239-49.

28. Flanagan $\mathrm{KL}$, Fink $\mathrm{AL}$, Plebanski M, Klein SL. Sex and gender differences in the outcomes of vaccination over the life course. Annu Rev Cell Dev Biol. 2017;33:577-99.

29. Vom Steeg LG, Flores-Garcia Y, Zavala F, Klein SL. Irradiated sporozoite vaccination induces sex-specific immune responses and protection against malaria in mice. Vaccine. 2019;37:4468-76

30. Witney AA, Doolan DL, Anthony RM, Weiss WR, Hoffman SL, Carucci DJ. Determining liver stage parasite burden by real time quantitative PCR as a method for evaluating pre-erythrocytic malaria vaccine efficacy. Mol Biochem Parasitol. 2001;118:233-45.

31. Schussek S, Groves PL, Apte SH, Doolan DL. Highly sensitive quantitative real-time PCR for the detection of Plasmodium liver-stage parasite burden following low-dose sporozoite challenge. PLOS ONE. 2013;8:e77811.

32. Billman ZP, Kas A, Stone BC, Murphy SC. Defining rules of CD8(+) T cell expansion against pre-erythrocytic Plasmodium antigens in sporozoiteimmunized mice. Malar J. 2016;15:238.

\section{Publisher's Note}

Springer Nature remains neutral with regard to jurisdictional claims in published maps and institutional affiliations.
Ready to submit your research? Choose BMC and benefit from:

- fast, convenient online submission

- thorough peer review by experienced researchers in your field

- rapid publication on acceptance

- support for research data, including large and complex data types

- gold Open Access which fosters wider collaboration and increased citations

- maximum visibility for your research: over $100 \mathrm{M}$ website views per year

At BMC, research is always in progress.

Learn more biomedcentral.com/submissions 